\title{
BLOCK CHAIN ENABLED INTERNET OF THINGS
}

\author{
Dr. D. Sivaganesan, \\ Professor, \\ Department of Computer Engineering, \\ PSG Institute of Technology and Applied Research, \\ Coimbatore, India. \\ Email Id: sivaganesan@psgitech.ac.in
}

\begin{abstract}
The internet of things constitutes for more and advanced opportunities paving way for a competitive advantage in numerous industrious in the prevailing and the upcoming markets. The internet of things and the technologies associated with it are not only creating alterations in the internetwork alone but also with the things that are connected to it. As the internet of things includes the generation and the analysis of the data as the important process, it finds difficulties in administering and protecting the data that are flowing from different levels. This in turn causes difficulties in the secure transmission of the information from its source to the authorized destination and the authorized person at the right time. So the above reasons bring in the capability of the block chain into the IOT, to improve the security as well as implant the transparency. The paper put forward a block chain enabled IOT for the pharmaceutical sector to elude the issue of counterfeit in the medicines.
\end{abstract}

Keywords: Internet of Things, Blockchain, Security, Transparency, Distributed network.

\section{INTRODUCTION}

The internetwork of things that is having a noted progress in the recent research and the industrial usage are liable getting affected by the security threats and the vulnerabilities [4] so as a protection to the vulnerability and the security issues block chain can be enabled into the IOT. The author Kshetri, in his paper describes the block chain as the "proof of digital ledger transactions" with the assurance to be shared among the persons involved in the transaction. [1] The basic concept the block chain relies on is the "tamper-proof storage of the approved transaction" [9] the block chain authorizes the devices incorporated into the internet of things to improve the security and in cultivate the transparency in the internet of things environment. The industrial development corporation predicts that by the dawn of the 2020 almost more than $20 \%$ of the internet of things deployment would be empowered with the block chain. [8] As the block chain provides distributed and a highly scalable ambience for the internet of things application, devices and the platforms. The fig .1 below shows the architecture of the IOT.

ISSN: 2582-418X (online)

Information Technology \& Digital World 
Journal of Information Technology and Digital World (2019)

Vol.01/ No. 01

Pages: $1-8$

https://www.irojournals.com/itdw/

DOI: https://doi.org/10.36548/jitdw.2019.1.001

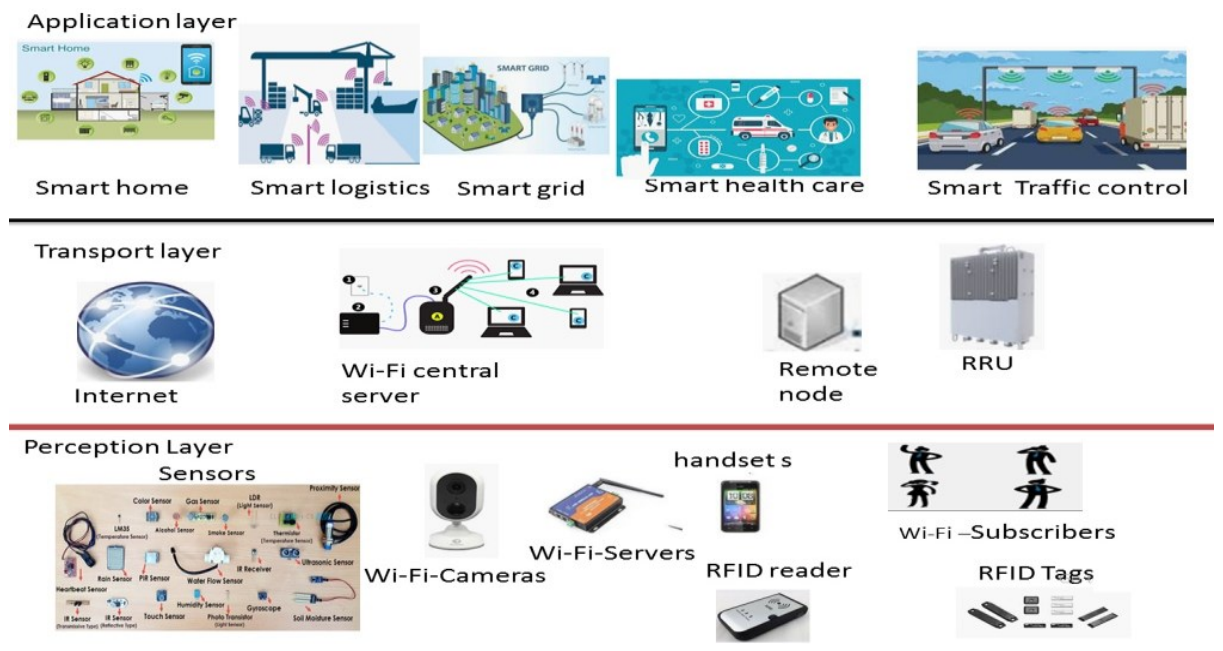

Fig .1 Architecture of the Internet of Things

Many financial sectors and multitudes of industries are planning to experience the capability of the block chain that is a distributed ledger. The Block chain and the IOT can be seen as the two sides of the coin as the blockchain provides transparency and security and the IOT ensures a smart functioning to the business or the organization or the industry associated with it. So the incorporation of the two would make a business, organization or the industry more efficient. The fig. 2 below shows the fundamental benefits in integrating the block chain with the internet of things.

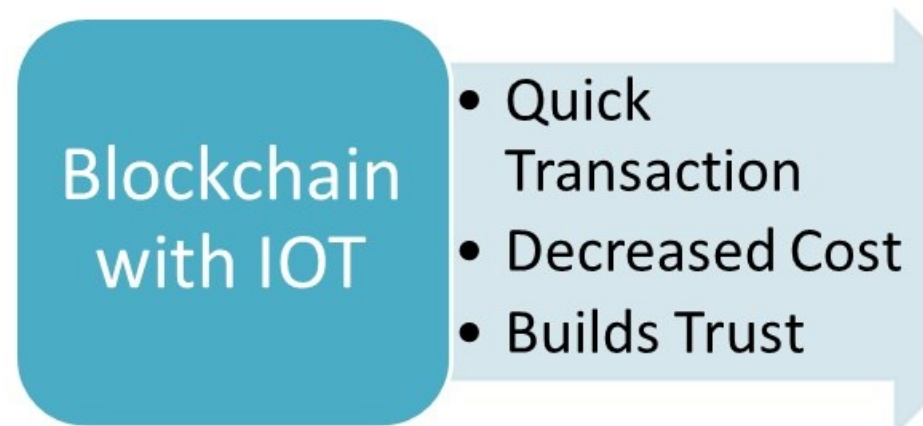

Fig .2 Benefits of Blockchain in IOT

Information Technology

$\&$

Digital World 
Journal of Information Technology and Digital World (2019)

Vol.01/ No. 01

Pages: $1-8$

https://www.irojournals.com/itdw/

DOI: https://doi.org/10.36548/jitdw.2019.1.001

The paper presents the block chain incorporated IOT for the pharmaceutical sector to elude the issue of counterfeit in the medicines.

The remaining paper is arranged with the related works in part 2, proposed work in part 3, results and discussion in part 4 and the conclusion in part 5.

\section{RELATED WORKS}

Kshetri, et al [1] the author presents the survey on the revealing the block chain capabilities for enhancing the security internet of things.

Conoscenti, et al [2] the author organizes a systematic review of the block chain presenting the prevailing uses of the "block chain" along with the counter measures for the research issues in the future.

Huckle, et al [3], the paper provides the beneficial's in the "shared economy" by incorporating the internetwork of things with the block chain.

Dorri, et al [4] the author mainly aims in providing system architecture for the IOT that is block chain based to avail a security and privacy to the internetwork of things.

Zhang, et al [5] the author proposes an elaborative discussion on the "block chain technology for the internet of things in the electric business models"

$\mathrm{Li}$, et al [6] the author puts forward a "unique unified energy block chain with a moderate cost for IIOT". The proffered system them in the paper utilizes the "credit card based payment scheme to uphold a "speedy and a frequent energy transaction.

Tian, Feng et al [7] the author presents the "supply chain traceability system for food safety based on hazard analysis and the critical control points, blockchain \& Internet of things". The paper modeled on the "concept of the BigchainDB to put in the gap in the decentralized systems at scale" and provides the issues in adapting to the block

ISSN: 2582-418X (online)

Information Technology Digital World 
Journal of Information Technology and Digital World (2019)

Vol.01/ No. 01

Pages: $1-8$

https://www.irojournals.com/itdw/

DOI: https://doi.org/10.36548/jitdw.2019.1.001

chain in the future.

Banafa, et al [8] the paper consolidates the benefits and the issues in the enabling the block chain for the internet of things. He presents the existing security measures as the mitigation for the risks found in the IOT and the put forward the block chain to be the optimized way of providing a secure communication through the IOT.

Samaniego, et al [9] the author exploits the "block chain as service for the IOT "as block chain efficient handles the configuration of the devices, the storage of the sensed data's and the payments. "He evaluates the block chain as service using the cloud and the fog as the hosting platform".

Pouraghily et al [10] the paper presents an architectural instruction for the internet of things enabled with the block chain and the ways of sharing them among a wide ecosystem simultaneously, to ensure a continuous access of them through the "block chain smart contracts".

Miraz, et al [11] and Mahdi $\mathrm{H}$ et al [12] to improvise the security of the internet of thing environment, the block chain technology that is the moving force of the bit-coin crypto currency system is adopted into the internet of things to heighten the level of security and the privacy in the internet of things. The paper also put forward a block-chain enriched IOT to increase the security of the internet of things. The fig. 3 below shows the processing steps involved in the Block chain.

\section{STIMULATE TRANSACTION}

VERIFICATION AND VALIDATION

NEW BLOCK CREATION
ADD BLOCK TO THE CHAIN
ISSN: 2582-418X (online)
Information Technology

\&

Digital World 
Journal of Information Technology and Digital World (2019)

Vol.01/ No. 01

Pages: $1-8$

https://www.irojournals.com/itdw/

DOI: https://doi.org/10.36548/jitdw.2019.1.001

Fig. 3 Blockchain Processing Steps [12]

Pachayappan et al [13] the author presents the internet of things based smart logistics design for the pharmaceutical sector by producing two plane architecture for the internet of things with the first layer formed for the radio frequency identification, and the second layer is the wireless sensor network.

\section{PROPOSED WORK}

The important work of the pharmaceutical industry is to take complete care of the medicines during the transportation, by maintaining a proper storage conditions and keeping note of the complete medicines that are transmitted to avoid the situations of risk. The pharmaceutical industry utilizes the methodology of the thermal and the refrigerator packaging for the transportation of the medicines. This is called the cold chain system. The cold chain system follows certain procedure such as the prepare, Store, transport and monitor, in the transportation of the medicine. It also takes care of the temperature that the medicines are to be maintained.

But the important issue that arises in the transportation of the medicines is the failure in the delivery of the information's during the transportation. So the IOT based smart logistics [13] was introduced for the pharmaceutical sector, to keep track of the movement of the shipment. But this did not provide the complete details of transportation and the happenings during the transportation of the medicines.

Nowadays as there are lots and lots of counterfeit and fake drugs that are produced in the market, this is also one of the issues that have to be addressed while transporting the drugs form on place to another, as there are chances of altering the good ones with the fake.

So the paper put forward the block chain combined IOT for the smart logistics of the pharmaceutical sector from one place to another. The blockchain technology with its traceable and the transparent nature helps in keeping track the shipment completely form its starting to its destination point and in order to have a credible transaction without the ISSN: 2582-418X (online) 
Journal of Information Technology and Digital World (2019)

Vol.01/ No. 01

Pages: $1-8$

https://www.irojournals.com/itdw/

DOI: https://doi.org/10.36548/jitdw.2019.1.001

third parties intervention, the proposed method also includes the smart contract that legally bonds the manufacturer the distributor and the dispenser

The fig.4 shows the proposed block chain enabled IOT for pharmaceutical sector to keep track of the shipment of the medicines.

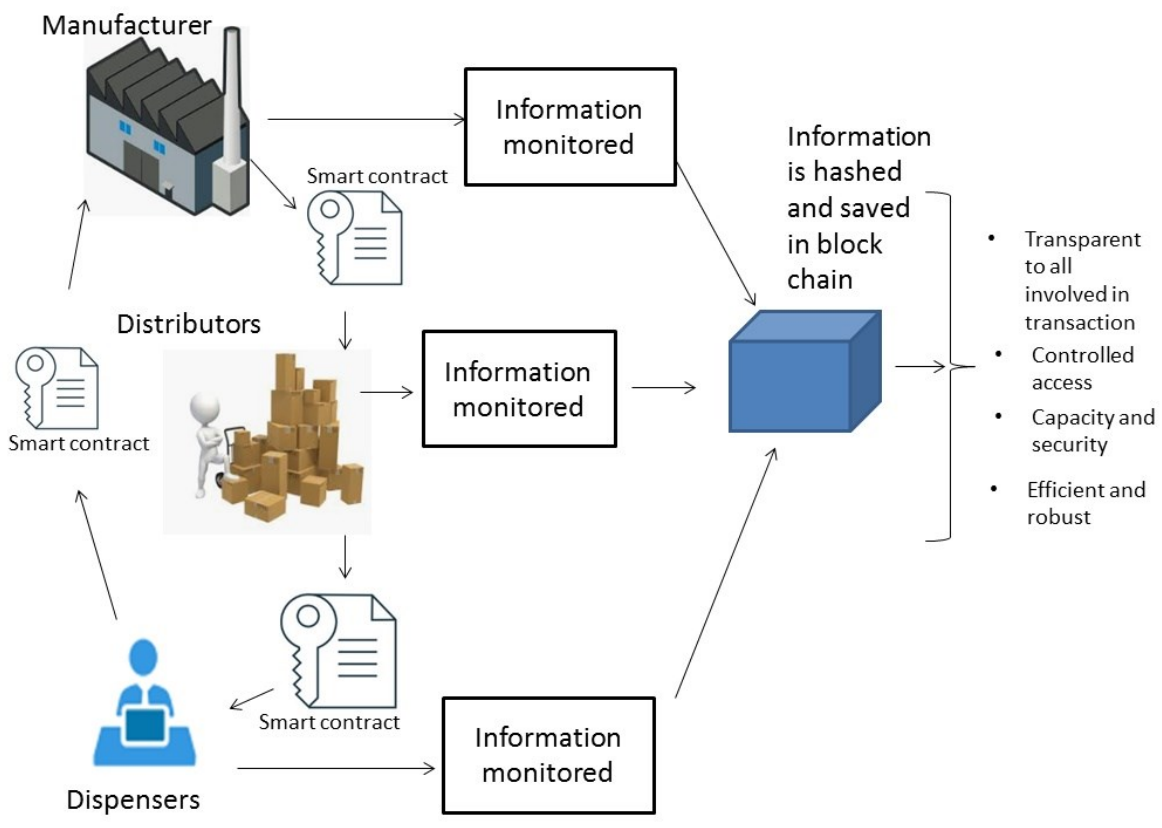

Fig.4 Block Chain Enabled Internet of Things

The block chain enabled IOT for the pharmaceutical sector decides the rules of the business, establishing the software standards for controlling the functions of the pharmacy utility such as the smart contract version, the financial management, capacity and security and system access/identity management and finally decides the node structure and the management. Each area involved in the shipment of the medicines is equipped with the sensor for tracking the status of the each operation that is done. The fig.4 shows the persons involved in the shipment of the medicine, the manufacture, who manufactures the drugs have sensors that completely tracks the details of the medicines manufactured, their medicines in stock, medicines expiry date and the composition of the medicines etc. in the same way the distributor sensor completely monitors the shipment and the shipment details finally the seller that is the dispenser holds a sensor that keeps track of the medicine available, newly arrived medicine, the ISSN: 2582-418X (online) 
Journal of Information Technology and Digital World (2019)

Vol.01/ No. 01

Pages: $1-8$

https://www.irojournals.com/itdw/

DOI: https://doi.org/10.36548/jitdw.2019.1.001

medicines sold and the medicine out of stock. The order placement, shipment and the selling of the medicines are verified using the smart contract that is maintained in between the person involved in the transaction, to avoid the receiving of the counterfeit drugs or fake drugs and the double payment for the medicine received etc.

The information tracked under the each person involved in the business is stored to the block chain. The blockchain being a distributed ledger makes the information stored in it unchangeable, time stamped and fully accessible/traceable, to all the persons who are involved in the transaction, for e.g. the manufacture details that are monitored when stored in the block chain would be visible of the other two who are involved in the business process. Similarly the data stored into the block chain by the distributor and the dispenser would also be visible to the all the persons who are involved in the transaction. This provides an easier way of supplying the medicines and receiving the payments in the block chain.

The Blockchain enabled internet of things with the smart contract allows a simplified payment process, a controlled user's access and reduces the counterfeiting drugs. The smart contract enabled between the each person in the transaction ensures the verification of the contract before and after reception of the medicines and the payments.

\section{RESULTS}

The paper presents the improvised security and transparency for the pharmaceutical sector to elude the counterfeit medicines in the market by introducing the Blockchain enabled IOT with the smart contract. This frame work could be used in any other supply chain to have highly secured and transparent transactions. The blockchain enabled IOT enables the persons involved in the transaction to have clear report of the each stage process and the smart contract enables the credible transactions compared to the smart logistics that is based only on the internet things. The blockchain in the stops the third person intervention in the pharmaceutical sector as well as he smart contract framing avoiding the leakage and the hacking of the information paving way for an improved security and transparency in the transaction for the

\section{CONCLUSION}

The paper proposing a novel methodology of transporting the medicine to reduce the availability of the counterfeit and the fake drugs in the market uses the block chain enabled internet of things for the pharmaceutical sector, in the transportation of the medicine and uses the smart contract to verify the credibility of the transaction. This allows the ISSN: 2582-418X (online)

Information Technology Digital World 
Journal of Information Technology and Digital World (2019)

Vol.01/ No. 01

Pages: $1-8$

https://www.irojournals.com/itdw/

DOI: https://doi.org/10.36548/jitdw.2019.1.001

manufacturer, distributor and the dispenser to have the complete details about the each stage of the transaction and ensures an enhanced security with the well-ordered user access and easy payment procedures the block chain combined internet of things enhances the efficiency of the system, and brings in the automation and robustness into the business process. In future the paper is to implement the blockchain enabled IOT with the smart contract into the agriculture to improve the supply chain of the agriculture.

\section{References}

[1] Kshetri, Nir. "Can blockchain strengthen the internet of things?." IT professional 19, no. 4 (2017): 68-72.

[2] Conoscenti, Marco, Antonio Vetro, and Juan Carlos De Martin. "Blockchain for the Internet of Things: A systematic literature review." In 2016 IEEE/ACS 13th International Conference of Computer Systems and Applications (AICCSA), pp. 1-6. IEEE, 2016.

[3] Huckle, Steve, Rituparna Bhattacharya, Martin White, and Natalia Beloff. "Internet of things, blockchain and shared economy applications." Procedia computer science 98 (2016): 461-466.

[4] Dorri, Ali, Salil S. Kanhere, and Raja Jurdak. "Blockchain in internet of things: challenges and solutions." arXiv preprint arXiv:1608.05187 (2016).

[5] Zhang, Yu, and Jiangtao Wen. "The IoT electric business model: Using blockchain technology for the internet of things." Peer-to-Peer Networking and Applications 10, no. 4 (2017): 983-994.

[6] Li, Zhetao, Jiawen Kang, Rong Yu, Dongdong Ye, Qingyong Deng, and Yan Zhang. "Consortium blockchain for secure energy trading in industrial internet of things." IEEE transactions on industrial informatics 14, no. 8 (2017): 3690-3700.

[7] Tian, Feng. "A supply chain traceability system for food safety based on HACCP, blockchain \& Internet of things." In 2017 International Conference on Service Systems and Service Management, pp. 1-6. IEEE, 2017.

[8] Banafa, Ahmed. "IoT and blockchain convergence: benefits and challenges." IEEE Internet of Things (2017).

[9] Samaniego, Mayra, and Ralph Deters. "Blockchain as a Service for IoT." In 2016 IEEE International Conference on Internet of Things (iThings) and IEEE Green Computing and Communications (GreenCom) and IEEE Cyber, Physical and Social Computing (CPSCom) and IEEE Smart Data (SmartData), pp. 433436. IEEE, 2016.

[10] Pouraghily, Arman, Md Nazmul Islam, Sandip Kundu, and Tilman Wolf. "Privacy in blockchain-enabled iot devices." In 2018 IEEE/ACM Third International Conference on Internet-of-Things Design and Implementation (IoTDI), pp. 292-293. IEEE, 2018.

[11] Miraz, Mahdi H., and Maaruf Ali. "Blockchain enabled enhanced IoT ecosystem security." In International ISSN: 2582-418X (online)

Information Technology Digital World 
Journal of Information Technology and Digital World (2019)

Vol.01/ No. 01

Pages: $1-8$

https://www.irojournals.com/itdw/

DOI: https://doi.org/10.36548/jitdw.2019.1.001

Conference for Emerging Technologies in Computing, pp. 38-46. Springer, Cham, 2018.

[12] Miraz, Mahdi H., and Maaruf Ali. "Applications of blockchain technology beyond cryptocurrency." arXiv preprint arXiv:1801.03528 (2018).

[13] Pachayappan, M., Nelavala Rajesh, and G. Saravanan. "Smart logistics for pharmaceutical industry based on Internet of Things (IoT)." In International Conference on Advances in Computational Intelligence and Communication (CIC 2016), pp. 31-36. 2016. 